Goldschmidt 2021 Abstract

https://doi.org/10.7185/gold2021.6249

\section{Biomineralization pathways in calcifying dinoflagellates: uptake, storage in MgCaP-rich bodies and formation of the shell}

\author{
ANNE JANTSCHKE ${ }^{1}$, ANDREAS SCHERTEL ${ }^{2}$, IDDO \\ PINKAS $^{3}$, LIA ADDADI ${ }^{3}$ AND STEVE WEINER ${ }^{3}$
}

${ }^{1}$ Institute of Geosciences, Johannes Gutenberg University

${ }^{2}$ Carl Zeiss Microscopy GmbH

${ }^{3}$ Weizmann Institute of Science

Presenting Author: jantschke@uni-mainz.de

During the last decades, significant progress has been made in understanding biomineral formation in model microalgae like diatoms and coccolithophores. In contrast, the mechanisms that control the intricate mineral construction in calcareous dinoflagellates are practically unknown. Our main objectives are to gain insight into their mineral architecture, subcellular structures that may play a role in biomineralization, and calcite morphogenesis. We investigate two representative members of calcareous dinoflagellates using cryo-electron microscopy (cryo SEM and cryo FIB SEM) in combination with various spectroscopic techniques (FT-IR, Raman, Fluorescence, EDS) enabling an investigation of cells as close as possible to the natural state [1].

Calcite formation occurs via multiple independent nucleation events inside the so-called outer matrix. Based on 2D and 3D cryo-electron microscopic datasets we show that individual calcite crystals grow with preferred orientation into a dense reticular network resulting in a highly regular, porous calcite shell (Fig. 1A).

In both species, we observed vacuoles containing crystalline inclusions using cryo-SEM. So far, crystalline deposits were assigned to calcite assuming that they are involved in shell formation. Surprisingly, using in situ Raman spectroscopic imaging we could identify these crystalline inclusions as anhydrous guanine in the biogenic $\beta$-form using their lowwavenumber Raman signature.

Interestingly, live-cell fluorescence imaging with Calcein-AM, cryo-sectioning and cryo-EDS show the presence of small $\mathrm{MgCaP}-$ rich mineral bodies within the same vacuolar enclosures (Fig. 1B). 3D cryo-FIB-SEM imaging of a calcifying cell shows a remarkably large number (353) of these bodies distributed in the cell volume. Out of these bodies, $52(\sim 15 \%)$ are located between the two inner organic layers of the outer matrix. We suggest that these $\mathrm{MgCaP}$-rich bodies are being secreted into the outer matrix and are part of a Ca-concentrating or transport mechanism.

Based on our results we developed a new dinoflagellate biomineralization model which includes the active uptake of $\mathrm{Ca}^{2+}$, temporary deposition in $\mathrm{MgCaP}$-precursor bodies, extrusion into the outer matrix and transformation into low $\mathrm{Mg}$ calcite [1].

[1] Jantschke, A., Pinkas, I., Schertel, A., Addadi, L., Weiner, S. (2020). Biomineralization pathways in calcifying dinoflagellates: uptake, storage in MgCaP-rich bodies and formation of the shell. Acta Biomaterialia 102, 427-439. DOI: 10.1016/j.actbio.2019.11.042

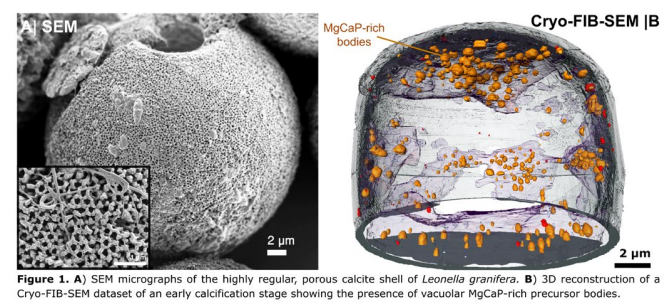

